\title{
Differential miRNA expression profiles in hepatocellular carcinoma cells and drug-resistant sublines
}

\author{
LIJUAN ZHUO $^{1}$, JINGFENG LIU ${ }^{2}$, BIN WANG $^{1}$, MEIQIN GAO $^{1}$ and AIMIN HUANG ${ }^{1}$ \\ ${ }^{1}$ Department of Pathology, School of Basic Medical Sciences, Fujian Medical University, Fuzhou 350004; \\ ${ }^{2}$ Division of Hepatobiliary Surgery, Hepatic Disease Center, The First Affiliated Hospital \\ of Fujian Medical University, Fuzhou 350005, P.R. China
}

Received July 20, 2012; Accepted October 8, 2012

DOI: $10.3892 /$ or.2012.2155

\begin{abstract}
Altered expression of miRNAs may contribute to multidrug resistance (MDR) in human cancers. This study investigated the association between miRNAs and MDR in five different drug-resistant hepatocellular carcinoma (HCC) cell sublines. The HCC Huh-7 cell line was treated with adramycin (ADM), cisplatin (DDP), carboplatin (CBP), mitomycin C (MMC) or vincristine (VCR) at increasing concentrations to develop drug-resistant sublines. The cell viability MTT assay was used to detect drug resistance. Five different drug-resistant HCC sublines, Huh-7/ADM, Huh-7/ CBP, Huh-7/DDP, Huh-7/MMC and Huh-7/VCR, were established. Cells that were resistant to one drug were also found to be resistant to the other drugs. miRNA microarrays were analyzed to identify differential miRNA expression profiles in these cell lines, and real-time PCR was used to validate miRNA microarray data. miRNA microarray analysis showed that there were 53 upregulated miRNAs in Huh-7/ADM, 56 in Huh-7/CBP, 58 in Huh-7/DDP, 58 in Huh-7/MMC and 49 in Huh-7/VCR, whereas there were 52 downregulated miRNAs in Huh-7/ADM, 50 in Huh-7/CBP, 41 in Huh-7/DDP, 55 in Huh-7/MMC and 56 in Huh-7/VCR. Moreover, 26 simultaneously upregulated and 25 simultaneously downregulated miRNAs were noted in the Huh-7/ADM, Huh-7/CBP, Huh-7/ DDP and Huh-7/MMC sublines compared to the parental Huh-7 cell line. In contrast, among these 51 upregulated and downregulated miRNAs, 12 miRNAs were upregulated and 13 miRNAs were downregulated in Huh-7/VCR. Upregulation of miR-27b, miR-181a, miR-146b-5p, miR-181d and miR-146a expression was verified using real-time RT-PCR in the parental and the five drug-resistant cell lines. In conclusion, the present study demonstrates that the differentially expressed miRNA
\end{abstract}

Correspondence to: Professor Aimin Huang, Department of Pathology, School of Basic Medical Sciences, Fujian Medical University, Fuzhou 350004, P.R. China

E-mail: aimin@mail.fjmu.edu.cn

Key words: hepatocellular carcinoma, chemotherapy, multidrug resistance, miRNA microarray, real-time PCR profiles in these five drug-resistant HCC sublines could be useful to further investigate the association of miRNA expression with drug resistance in $\mathrm{HCC}$.

\section{Introduction}

Hepatocellular carcinoma (HCC) is a significant worldwide health problem accounting for more than 750,000 new cases and more than 690,000 cancer-related deaths in 2011 (1), in which half of these cases and deaths were estimated to have occurred in China alone. HCC incidence rates are increasing in many parts of the world, including the US and Central Europe. To date, surgery is still the primary treatment for HCC. However, there are not many patients who are eligible to receive surgery due to a variety of reasons, such as unresectable tumors, distant tumor metastasis, insufficient hepatic function and poor health conditions. Moreover, HCC has a high recurrence rate after resection. Thus, neoadjuvant therapy is usually used to treat HCC patients. For example, transarterial chemoembolization (TACE), which is an important neoadjuvant treatment against $\mathrm{HCC}$, has been extensively used to delay $\mathrm{HCC}$ progression in the clinic and to improve the prognosis of HCC patients. Chemotherapeutical drugs, such as adriamycin (ADM), mitomycin C (MMC), vincristine (VCR), 5-fluorouracil (5-FU), cisplatin (DDP) and carboplatin (CBP), are frequently used in HCC treatment $(2,3)$. Among them, ADM is an antibiotic that eliminates tumor cells by preventing RNA biosynthesis. MMC, also an antibiotic, is able to directly destroy DNA and prevent DNA replication. Both DDP and CBP confer antitumor effects through inhibition of DNA biosynthesis and replication. In contrast, VCR targets the microtubules in cells, interferes with protein metabolism and inhibits activity of RNA polymerase and synthesis of plasmalemma adipoid. In the clinic, two or three drugs are combined together with TACE. However, multidrug resistance (MDR) prevents successful long-term use of chemotherapy. MDR mechanisms are usually complicated and many factors, such as P-glycoprotein (P-gp), resistance-associated protein (MRP), lung resistance protein (LRP), glutathione $\mathrm{S}$-transferase (GST), cyclooxygenase 2 (COX-2), nuclear factor- $\kappa \mathrm{B}(\mathrm{NF}-\kappa \mathrm{B})$ (4-9), have been found to participate in MDR.

More recently, it has been shown that altered expression of miRNAs plays an important role in MDR (10). miRNAs 
are small non-coding RNA molecules of 20-23 nucleotides in length and regulate a variety of biological processes. miRNAs inhibit mRNA translation of target genes through imperfect base pairing with the 5'- or 3'-untranslated region of the target mRNAs. Previous studies have underlined the involvement of miRNAs in drug resistance (11-18). Different miRNAs have been shown to be important in the mediation of chemosensitivity or chemoresistance in different cancer types, which occurs through the regulation of MDR- or cellgrowth-related protein expression. Expression of miR-326 was found to downregulate MRP-1 expression and sensitize tumor cells to VP-16 and doxorubicin (13). Overexpression of miR-122 modulated HCC sensitivity to chemotherapeutic drugs through downregulation of MDR-related genes MDR-1, GST- $\pi$, MRP, antiapoptotic gene Bcl-w and cell cycle-related gene cyclin-B1 (15). Expression of miR-34a can negatively regulate, at least in part, resistance of colorectal cancer DLD-1 cells to 5-FU through targeting Sirt1 and E2F3 genes (19). In contrast, stable transfection of miR-21 induced drug resistance in K562 cells, while suppression of miR-21 in K562/DNR cells enhanced DNR cytotoxicity (20). miR-214 induces cell survival and DDP resistance primarily through targeting the PTEN/Akt pathway (21). miR-328 targets ABCG2 3'-UTR and, consequently, controls ABCG2 protein expression and influences drug disposition in human breast cancer cells (22). However, upregulation of miR-138 reverses resistance of both P-gp-related and P-gp-non-related drugs in HL-60/VCR cells and promotes adriamycin-induced apoptosis (16). Although these studies provide insightful information regarding miRNA-mediated MDR in human cancers, a complete profile of miRNA-mediated MDR is needed to systematically understand the role of miRNAs in MDR in HCC. Therefore, in this study, we first established five chemotherapeutical drug-resistant HCC cell sublines, Huh-7/ADM, Huh-7/DDP, Huh-7/CBP, Huh-7/MMC and Huh-7/VCR. We then subsequently profiled altered miRNA expression in these sublines in comparison to the parental HCC cell line using miRCURY ${ }^{\mathrm{TM}}$ LNA array (v. 16.0).

\section{Materials and methods}

Cell line and culture. The human HCC Huh-7 cell line was obtained from the Cell Bank of the Chinese Academy of Sciences (Shanghai, China) and cultured in RPMI-1640 medium (Hyclone, Logan, UT, USA) supplemented with $10 \%$ fetal bovine serum (FBS) (Gibco-BRL; Invitrogen Life Technologies, Carlsbad, CA, USA) in a humidified incubator at $37^{\circ} \mathrm{C}$ with $5 \%$ carbon dioxide.

Generation of drug-resistant HCC cell sublines. The different drug-resistant HCC cell sublines were established by exposing the parental Huh-7 cells to increased concentrations of various chemotherapeutic drugs. Briefly, Huh-7 cells were inoculated in a 10-ml cell culture flask and cultivated for $72 \mathrm{~h}$ in culture medium containing a low concentration of drugs $(0.02 \mu \mathrm{g} / \mathrm{ml}$ ADM, $0.02 \mu \mathrm{g} / \mathrm{ml} \mathrm{CBP,} 0.0375 \mu \mathrm{g} / \mathrm{ml}$ DDP, $0.0015 \mu \mathrm{g} / \mathrm{ml}$ MMC, or $0.01 \mu \mathrm{g} / \mathrm{ml} \mathrm{VCR).} \mathrm{Subsequently,} \mathrm{the} \mathrm{cells} \mathrm{were}$ continuously cultured without drug exposure for $\sim 2$ weeks. When cell growth was in the logarithmic phase, the cells were collected and re-inoculated in a 10 -ml culture flask in culture medium containing the above-mentioned drugs at an elevated concentration (1.5- to 2-fold of the previous dose) or at a previous concentration. This procedure was repeated until the cells exhibited stable growth and proliferation in a culture medium with $4.0 \mu \mathrm{g} / \mathrm{ml}$ ADM, $0.4 \mu \mathrm{g} / \mathrm{ml} \mathrm{CBP}, 0.6 \mu \mathrm{g} / \mathrm{ml}$ DDP, $0.1 \mu \mathrm{g} / \mathrm{ml} \mathrm{MMC} \mathrm{or} 4.0 \mu \mathrm{g} / \mathrm{ml} \mathrm{VCR}$. A period of $\sim 10-15$ months was required to establish these drug-resistant HCC cell sublines. The level of drug resistance was determined using the 3-(4,5-dimethylthiazol-2-yl)-2,5-diphenyltetrazolium bromide (MTT) assay.

Cell viability MTT assay. Exponentially growing cells were seeded at 6,000-10,000 cells/well in 96-well plates with $100 \mu \mathrm{l}$ of culture medium/well and incubated for $6 \mathrm{~h}$. The cells were then exposed to different concentrations of the drugs for $65 \mathrm{~h}$. At the end of the experiments, $20 \mu \mathrm{l}$ of MTT $(5 \mathrm{mg} / \mathrm{ml}$ in PBS) was added in each well, and the cells were cultured for an additional $4 \mathrm{~h}$ for formation of formazan crystals. Subsequently, $150 \mu 1$ of DMSO was added to each well to dissolve the crystals. The values of the optical density at $570 \mathrm{~nm}$ were then measured using a microplate ELISA reader. Each experiment was performed in triplicate and repeated trice. Resistance factors (RF) were calculated by dividing the $\mathrm{IC}_{50}$ value (drug concentration resulting in 50\% reduction in absorbance compared with the control) of the drug-resistant cells with that of the parental control cells.

miRNA microarray analysis. Total cellular RNA was isolated using TRIzol (Invitrogen Life Technologies) and then cleaned using the miRNeasy Mini kit (Qiagen) according to the manufacturer's instructions. RNA quality and quantity were measured using a Nanodrop spectrophotometer (ND-1000; Nanodrop Technologies, Wilmington, DE, USA), and RNA integrity was determined using gel electrophoresis. Subsequently, the miRCURY ${ }^{\mathrm{TM}}$ Hy3/Hy5 Power labeling kit (Exiqon, Vedbaek, Denmark) was used to label the RNA as probes according to the manufacturer's guideline. Next, the Hy3TM-labeled RNA samples were hybridized on miRCURY ${ }^{\mathrm{TM}}$ LNA array (v. 16.0) (Exiqon) slides according to the array manual. The slides were then washed three times with wash buffer (Exiqon) and dried using centrifugation for $5 \mathrm{~min}$ at $400 \mathrm{rpm}$. Finally, the slides were scanned using the Axon GenePix 4000B microarray scanner (Axon Instruments, Foster City, CA, USA).

The scanned images were imported into GenePix Pro 6.0 software (Axon Instruments) for grid alignment and data extraction. Replicated miRNAs were averaged and miRNAs with intensities $>50$ in all the samples were chosen to calculate a normalization factor. Expressed data were then normalized using the median normalization. Differentially expressed miRNAs were then identified according to the fold changes and intensities, and hierarchical clustering was performed using MEV software (v. 4.6, TIGR).

Quantitative reverse transcription-polymerase chain reaction ( $q R T-P C R)$. Total cellular RNA samples from the cells were reversely transcribed into cDNA using a Universal cDNA synthesis kit (Exiqon) according to the manufacturer's instructions. Real-time PCR amplification was performed on an ABI 7500 Real-Time PCR system with the DNA-binding 


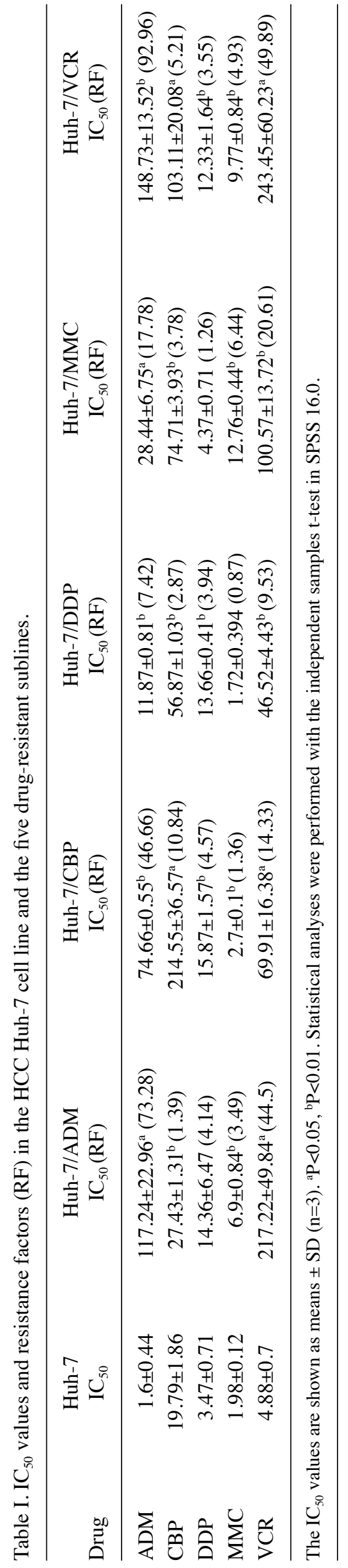

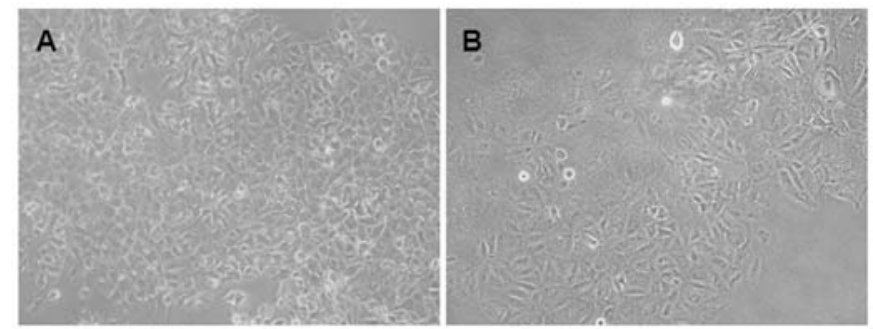
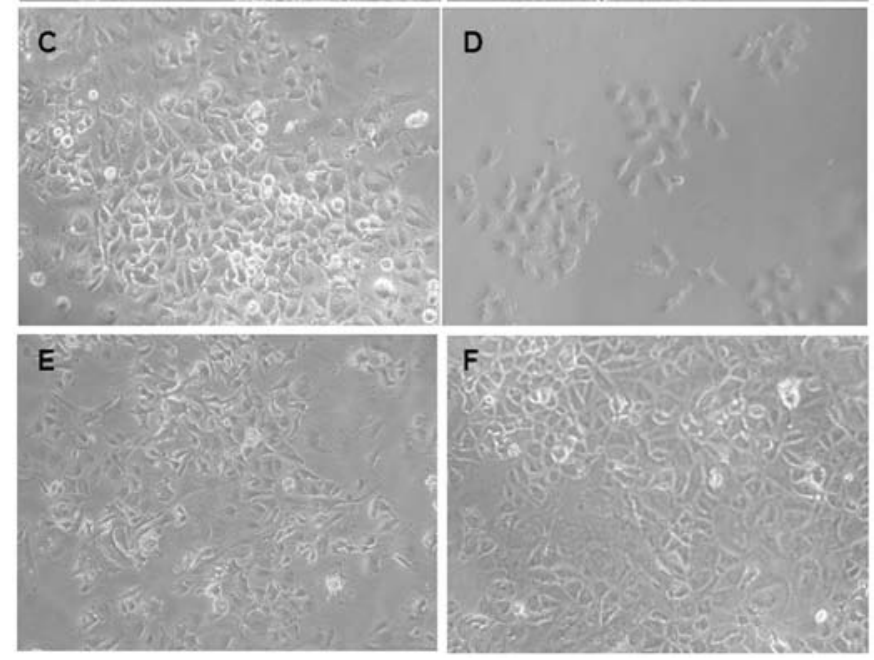

Figure 1. Changes in cell morphology of the five drug-resistant HCC sublines. (A) Huh-7; (B) Huh-7/ADM; (C) Huh-7/CBP; (D) Huh-7/DDP; (E) Huh-7/MMC; (F) Huh-7/VCR. The cell morphology of the drug-resistant cells reveals larger and more cellular pleomorphism than that of the parental Huh-7 cells, although their shapes are polygon and similar to the Huh-7 cells. In contrast, some cells have long pseudopodia (magnification, $\mathrm{x} 200$ ).

dye technique (SYBR Green) according to the manufacturer's instructions. The primers for the different miRNAs and PCR reagents were purchased from Exiqon. U6 snRNA was used as the reference gene, and $2^{-\Delta \Delta c t}$ was used to calculate the expression levels of miRNAs in the drug-resistant HCC cell sublines compared to the parental Huh-7 cell line.

\section{Results}

Establishment of different chemotherapeutical drug-resistant HCC cell sublines. In this study, we first established drugresistant HCC cell sublines by treating HCC Huh-7 cells with increasing doses of ADM, DDP, CBP, MMC and VCR for 10-15 months. The drug-resistant concentration of ADM was $4.0 \mu \mathrm{g} / \mathrm{ml}$, CBP was $0.4 \mu \mathrm{g} / \mathrm{ml}$, DDP was $0.6 \mu \mathrm{g} / \mathrm{ml}$, MMC was $0.1 \mu \mathrm{g} / \mathrm{ml}$ and VCR was $4.0 \mu \mathrm{g} / \mathrm{ml}$. The HCC cell sublines were named Huh-7/ADM, Huh-7/DDP, Huh-7/CBP, Huh-7/ MMC and Huh-7/VCR, respectively. The $\mathrm{IC}_{50}$ values and $\mathrm{RF}$, which are shown in Table I, indicated that these drug-resistant sublines were not only resistant to the treatment drug, but also resistant to the other drugs. Under an inverted microscope, although the drug-resistant cells were similar to the parental Huh-7 cells, the drug-resistant cells grew slowly as colonies and most of the cell lines had an enlarged cell body (Fig. 1).

Differential expression of miRNAs in the drug-resistant HCC cell sublines. To profile differential expression of various miRNAs in the drug-resistant HCC sublines, we performed 

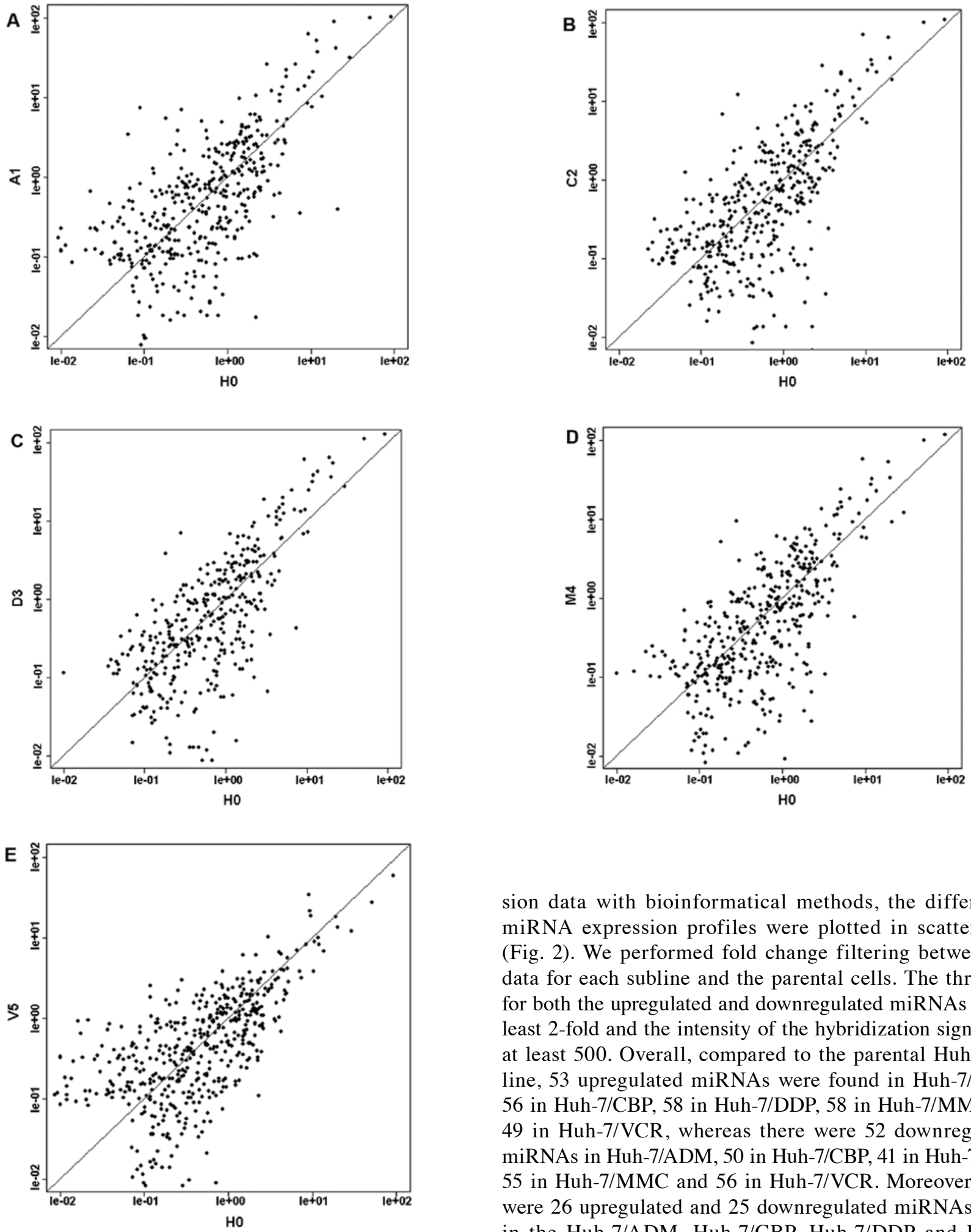

Figure 2. miRNA expression profiles between the five drug-resistant cell sublines and their parental Huh-7 cell line. The axes of the scatter-plot are the normalized signal values (ratio scale). The variation in miRNA expression levels in the drug-resistant sublines compared to Huh-7 cells were similar for Huh-7/ADM, Huh-7/CBP, Huh-7/DDP and Huh-7/MMC. H0, Huh-7; A1, Huh-7/ADM; C2, Huh-7/CBP; D3, Huh-7/DDP; M4, Huh-7/MMC; V5, Huh-7/VCR. (A) $\mathrm{R}_{\mathrm{Al}-\mathrm{H} 0}=0.8277$, (B) $\mathrm{R}_{\mathrm{C} 2-\mathrm{H} 0}=0.8451$, (C) $\mathrm{R}_{\mathrm{D} 3-\mathrm{H} 0}=0.9093$, (D) $\mathrm{R}_{\mathrm{M} 4-\mathrm{H} 0}=0.8964$, (E) $\mathrm{R}_{\mathrm{V} 5-\mathrm{H} 0}=0.8647$.

miRNA microarray analysis in these sublines, as well as in the parental HCC Huh-7 cells. After normalizing the expres-

sion data with bioinformatical methods, the differential miRNA expression profiles were plotted in scatter-plots (Fig. 2). We performed fold change filtering between the data for each subline and the parental cells. The threshold for both the upregulated and downregulated miRNAs was at least 2-fold and the intensity of the hybridization signal was at least 500. Overall, compared to the parental Huh-7 cell line, 53 upregulated miRNAs were found in Huh-7/ADM, 56 in Huh-7/CBP, 58 in Huh-7/DDP, 58 in Huh-7/MMC and 49 in Huh-7/VCR, whereas there were 52 downregulated miRNAs in Huh-7/ADM, 50 in Huh-7/CBP, 41 in Huh-7/DDP, 55 in Huh-7/MMC and 56 in Huh-7/VCR. Moreover, there were 26 upregulated and 25 downregulated miRNAs noted in the Huh-7/ADM, Huh-7/CBP, Huh-7/DDP and Huh-7/ MMC sublines. However, among these 51 upregulated and downregulated miRNAs, 12 miRNAs were upregulated and 13 miRNAs were downregulated in the Huh-7/VCR subline. We then performed a hierarchical clustering analysis for these miRNAs (Fig. 3). Fig. 4 shows the normalized value of these differentially expressed miRNAs between Huh-7 cells and the Huh-7/ADM, Huh-7/CBP, Huh-7/DDP, Huh-7/MMC and Huh-7/VCR sublines.

Validation of several differentially expressed miRNAs in HCC cells. We validated the microarray data using real-time RT-PCR 

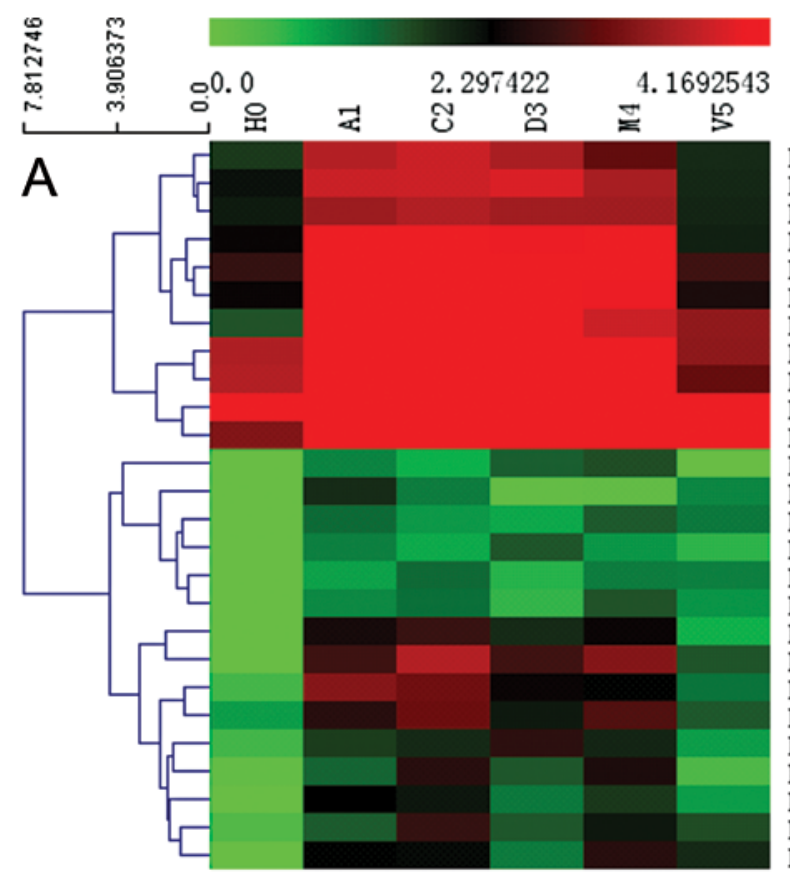

hsa-miR-3607-3p

hsa-miR-1274a

hsa-miRPlus-A1015

hsa-miR-3653

hsa-miR-1260

hsa-miR-3647-3p

hsa-miR-194

hsa-miR-1246

hsa-miR-1280

hsa-miR-1290

hsa-miR-22

hsa-miR-138-2*

hsa-miR-186*

hsa-miR-23a

hsa-miR-26b

hs $a-m i R-27 b$

hsa-miR-181d

hsa-miR-146b-5p

hsa-miR-146a

hsa-miR-192

hsa-miR-16

hsa-miR-29a

hsa-miR-15a

hsa-miR-181a

hsa-miR-30c

hsa-miR-30b
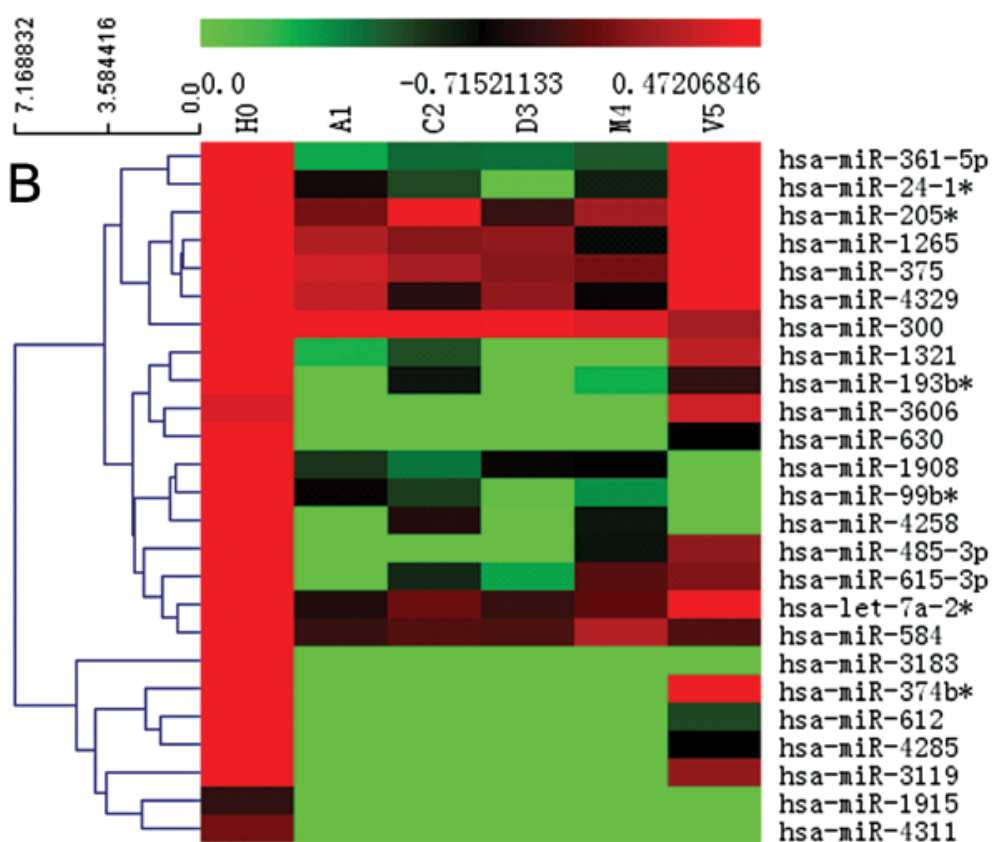

Figure 3. Hierarchical clustering analysis of the miRNA microarray data. (A) Analyses of the 26 upregulated miRNAs in Huh-7/ADM, Huh-7/CBP, Huh-7/DDP and Huh-7/MMC. (B) Analyses of the 25 downregulated miRNAs in Huh-7/ADM, Huh-7/CBP, Huh-7/DDP and Huh-7/MMC. The heat map diagram shows the results of the two-way hierarchical clustering analysis of miRNA expression levels and the cell lines. Each row represents an miRNA and each column represents a cell line. The miRNA-clustering tree is shown on the left and the cell line-clustering tree is at the top. The color scale shown at the top illustrates the relative expression level of an miRNA in a certain slide. A red color represents a high relative expression level and a green color represents a low relative expression level. H0, Huh-7; A1, Huh-7/ADM; C2, Huh-7/CBP; D3, Huh-7/DDP; M4, Huh-7/MMC; V5, Huh-7/VCR.

analysis. We chose the five most significantly expressed miRNAs (miR-27b, miR-181a, miR-146b-5p, miR-181d and miR-146a) to be verified in Huh-7, Huh-7/ADM, Huh-7/CBP, Huh-7/DDP, Huh-7/MMC and Huh-7/VCR cells. Our qRT-PCR data confirmed the microarray data (Table II). Table II also lists potential target genes of these miRNAs.

\section{Discussion}

In the present study, we first established five different chemotherapeutic drug-resistant HCC cell sublines and then profiled the altered miRNA expression in these sublines in comparison to the parental HCC cell line using miRCURY ${ }^{\mathrm{TM}}$ LNA array (v. 16.0). We found that cells that were resistant to one drug were also resistant to the other drugs. miRNA microarray data revealed 53 upregulated miRNAs in Huh-7/ADM, 56 in Huh-7/CBP, 58 in Huh-7/DDP, 58 in Huh-7/MMC and 49 in the Huh-7/VCR subline. In contrast, there were 52 downregulated miRNAs in Huh-7/ADM, 50 in Huh-7/CBP, 41 in Huh-7/DDP, 55 in Huh-7/MMC and 56 in Huh-7/VCR. In total, there were 26 simultaneously upregulated and 25 simultaneously downregulated miRNAs in Huh-7/ADM, Huh-7/CBP, Huh-7/DDP 

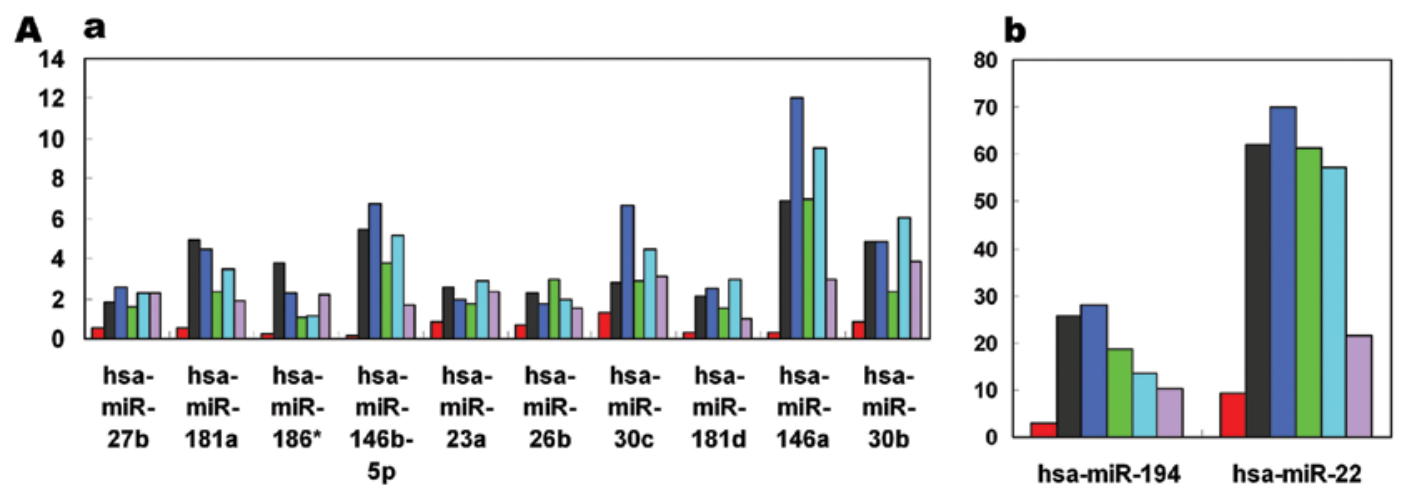

$\mathbf{B}$

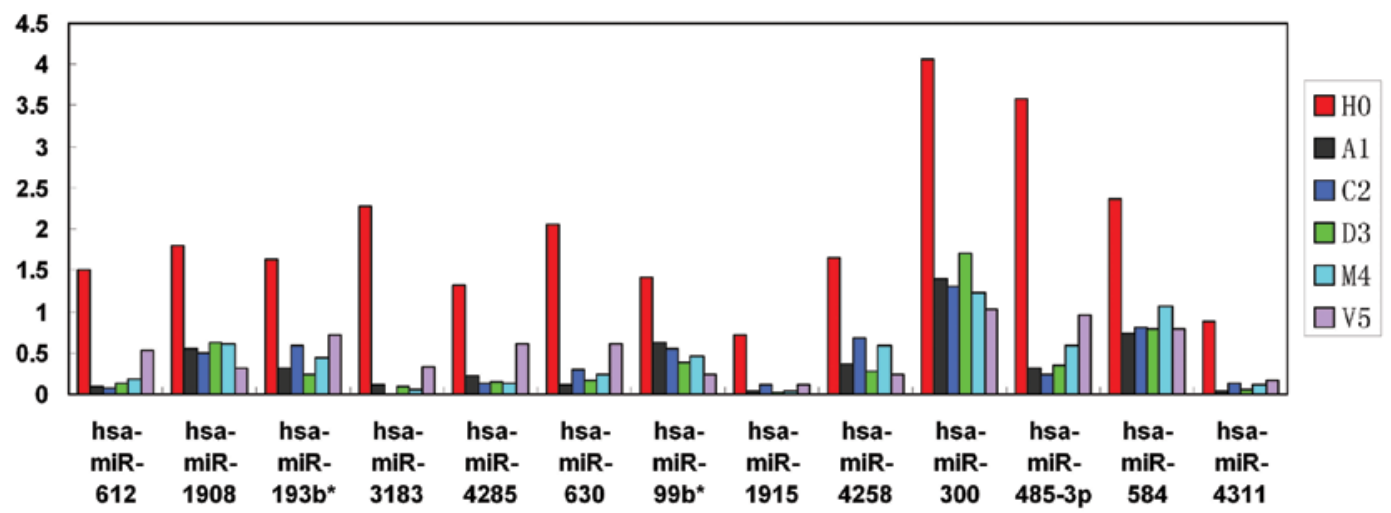

Figure 4. Analyses of the miRNAs exhibiting differential expression in the Huh-7 cell line and the drug-resistant sublines. (Aa and b) Normalized values of the simultaneously upregulated miRNAs in the five drug-resistant sublines. (B) Normalized values of the simultaneously downregulated miRNAs in the five drug-resistant cell sublines. H0, Huh-7; A1, Huh-7/ADM; C2, Huh-7/CBP; D3, Huh-7/DDP; M4, Huh-7/MMC; V5, Huh-7/VCR.

Table II. Confirmation of miRNA expression levels in the five drug-resistant sublines.

Fold changes (microarray, real-time PCR ${ }^{\mathrm{a}}$ )

\begin{tabular}{|c|c|c|c|c|c|c|c|c|c|}
\hline \multirow[b]{2}{*}{ miRNA } & \multicolumn{2}{|c|}{$\mathrm{A} 1 / \mathrm{H} 0$} & \multicolumn{2}{|c|}{$\mathrm{C} 2 / \mathrm{H} 0$} & \multicolumn{2}{|c|}{$\mathrm{D} 3 / \mathrm{H} 0$} & \multicolumn{2}{|c|}{$\mathrm{M} 4 / \mathrm{H} 0$} & \multirow[b]{2}{*}{ Target genes } \\
\hline & Array & $\mathrm{qPCR}$ & Array & qPCR & Array & $\mathrm{qPCR}$ & Array & qPCR & \\
\hline $\operatorname{miR}-27 b$ & 3.54 & 20.13 & 5.02 & 7.48 & 3.04 & 1.72 & 4.45 & 4.56 & BAX, P53, FoxO1, KRAS \\
\hline miR-181a & 9.98 & 22.32 & 8.99 & 12.41 & 4.77 & 2.17 & 7.02 & 6.45 & PTEN, KRAS, RB1 \\
\hline $\operatorname{miR}-146 b-5 p$ & 29.84 & 35.47 & 36.96 & 10.14 & 20.75 & 4.28 & 28.15 & 3.85 & TRAF6, PTGS2 \\
\hline $\operatorname{miR}-181 d$ & 7.08 & 6.97 & 8.20 & 3.86 & 4.97 & 2.02 & 9.80 & 3.19 & PTEN, KRAS, RB1 \\
\hline miR-146a & 24.44 & 441.13 & 42.60 & 96.45 & 24.57 & 12.95 & 33.69 & 50.66 & TRAF6, PTGS2 \\
\hline
\end{tabular}

H0, Huh-7; A1, Huh-7/ADM; C2, Huh-7/CBP; D3, Huh-7/DDP; M4, Huh-7/MMC; V5, Huh-7/VCR. ${ }^{\mathrm{a}}<<0.001$ for all differences. Information regarding the miRNA target genes was obtained from http://www.microrna.org/.

and Huh-7/MMC. Among these 51 altered miRNAs, 12 were upregulated and 13 miRNAs were downregulated in the Huh-7/VCR subline. We chose miR-27b, miR-181a, miR146b-5p, miR-181d and miR-146a to verify the results with real-time RT-PCR. This study profiled altered miRNA expression in the five most commonly used drug-induced HCC resistant sublines, which provides useful information for further investigation of miRNA-mediated drug resistance in HCC.

Generally, to study the mechanisms responsible for tumor multidrug resistance, three methods are usually used to establish a multidrug-resistant tumor cell line. These methods include the induction of drug resistant cell lines in vitro, MDR gene transfection, or the induction of drug resistance with a nude mouse implanted tumor model. To induce tumor cell MDR in vitro, two different cell culture methods are used: the drug concentration incremental gradient method and the highconcentration intermittent drug-induced method (23). In the present study, we established drug-resistant cell sublines using the drug concentration incremental gradient method. The drug-resistant cell sublines were resistant not only to the drug 
used to induce the subline, but also to the other drugs, which is characteristic of MDR. These data provide the foundation to pursue subsequent research concerning the altered expression of miRNAs in these sublines.

A large number of miRNAs with altered expression were noted in these sublines compared to the parental HCC cell line. Several of the miRNAs, such as the miR-15/16 family (14), miR-30c (24,25), the miR-181 family $(18,26,27)$, miR-23a (28), miR-192 (29), miR-27b (12), miR-194 (12,29), miR-22 (12), miR-29a (12,30), miR-146a (29) and the let-7 family $(31,32)$ have been previously reported to be related to chemoresistance in different types of cancers. Specifically, miR-15b and miR-16 play a role in the development of MDR in gastric cancer through modulation of apoptosis by targeting Bcl-2 expression (14), while miR-30c was found to be downregulated in various chemoresistant tumor cell lines (23). Moreover, miR-181d was upregulated in two docetaxel-induced head and neck squamous cell cancer MDR cell lines (27), and miR-23a was shown to be an upstream regulator of TOP2B that mediated cisplatin chemoresistance in tongue squamous cell carcinoma cell lines (28). Furthermore, miR-192 was found to target dihydrofolate reductase, a key enzyme in folate metabolism that influences sensitivity in colorectal cancer cell lines when treated with 5-FU (29). In addition, breast cancer MCF-7/DOX cells resistant to DOX treatment were associated with the increased expression of miR-22, miR-29a, miR-194 and miR-132 (12). miR-let-7a was downregulated in the NCI-60 cell line, which is sensitive to cyclophosphamide (31). Our current data revealed 12 simultaneously upregulated and 13 downregulated miRNAs in the five HCC drug-resistant cell sublines. Further investigation of these 25 miRNAs may help elucidate HCC cell MDR to these five drugs.

In addition, we used a SYBR-Green miRNA real-time qPCR analysis to validate the expression of miR-27b, miR-181a, miR-146b-5p, miR-181d and miR-146a in the drugresistant HCC cell sublines. miR-27b, which is localized at chromosome $\mathrm{q} 22.32$, has been reported to play a role in cell growth, tumor progression and the inflammatory response (33-36). miRNA-146a/b has been shown to be involved in the modulation of inflammatory responses (37), immune responses (38) and suppression of tumor metastasis (39-41). miR-181a was reported to be associated with patient prognosis $(42,43)$. Finally, miR-181a and mir-181d (44) have been shown to have antitumor activity (43). However, their role in HCC MDR will be further investigated in our next study.

\section{Acknowledgements}

This study was supported in part by a grant from the Department of Education, Fujian Province, China (\#JA11114). We would like to thank Ms. Ren-Nan of Fujian Medical University, School of Public Health for her technical assistance in the qRT-PCR analyses.

\section{References}

1. Jemal A, Bray F, Center MM, Ferlay J, Ward E and Forman D: Global cancer statistics. CA Cancer J Clin 61: 69-90, 2011.

2. Lee JO, Kim DY, Lim JH, et al: Palliative chemotherapy for patients with recurrent hepatocellular carcinoma after liver transplantation. J Gastroenterol Hepatol 24: 800-805, 2009.
3. Tomuleasa C, Soritau O, Fischer-Fodor E, et al: Arsenic trioxide plus cisplatin/interferon alpha-2b/doxorubicin/capecitabine combination chemotherapy for unresectable hepatocellular carcinoma. Hematol Oncol Stem Cell Ther 4: 60-66, 2011.

4. Walsh N, Larkin A, Kennedy S, et al: Expression of multidrug resistance markers ABCB1 (MDR-1/P-gp) and ABCC1 (MRP-1) in renal cell carcinoma. BMC Urol 9: 6, 2009.

5. Shi H, Lu D, Shu Y, Shi W, Lu S and Wang K: Expression of multidrug-resistance-related proteins P-glycoprotein, glutathione-S-transferases, topoisomerase-II and lung resistance protein in primary gastric cardiac adenocarcinoma. Cancer Invest 26: 344-351, 2008 .

6. Mansilla S, Rojas M, Bataller M, Priebe W and Portugal J: Circumvention of the multidrug-resistance protein (MRP-1) by an antitumor drug through specific inhibition of gene transcription in breast tumor cells. Biochem Pharmacol 73: 934-942, 2007.

7. Filomeni G, Turella P, Dupuis ML, et al: 6-(7-Nitro-2,1,3-benzoxadiazol-4-ylthio)hexanol, a specific glutathione S-transferase inhibitor, overcomes the multidrug resistance (MDR)-associated protein 1-mediated MDR in small cell lung cancer. Mol Cancer Ther 7: 371-379, 2008.

8. Ronaldson PT, Ashraf T and Bendayan R: Regulation of multidrug resistance protein 1 by tumor necrosis factor alpha in cultured glial cells: involvement of nuclear factor-kappaB and c-Jun N-terminal kinase signaling pathways. Mol Pharmacol 77: 644-659, 2010.

9. Liu B, Qu L and Tao H: Cyclo-oxygenase 2 up-regulates the effect of multidrug resistance. Cell Biol Int 34: 21-25, 2010.

10. Ma J, Dong C and Ji C: MicroRNA and drug resistance. Cancer Gene Ther 17: 523-531, 2010.

11. Feng DD, Zhang H, Zhang P, et al: Down-regulated miR-331-5p and miR-27a are associated with chemotherapy resistance and relapse in leukaemia. J Cell Mol Med 15: 2164-2175, 2011.

12. Kovalchuk O, Filkowski J, Meservy J, et al: Involvement of microRNA-451 in resistance of the MCF-7 breast cancer cells to chemotherapeutic drug doxorubicin. Mol Cancer Ther 7: 2152-2159, 2008

13. Liang $\mathrm{Z}, \mathrm{Wu} \mathrm{H}, \mathrm{Xia} \mathrm{J}$, et al: Involvement of miR-326 in chemotherapy resistance of breast cancer through modulating expression of multidrug resistance-associated protein 1 . Biochem Pharmacol 79: 817-824, 2010.

14. Xia L, Zhang D, Du R, et al: miR-15b and miR-16 modulate multidrug resistance by targeting BCL2 in human gastric cancer cells. Int J Cancer 123: 372-379, 2008.

15. Xu Y, Xia F, Ma L, et al: MicroRNA-122 sensitizes HCC cancer cells to adriamycin and vincristine through modulating expression of MDR and inducing cell cycle arrest. Cancer Lett 310: 160-169, 2011.

16. Zhao X, Yang L, Hu J and Ruan J: miR-138 might reverse multidrug resistance of leukemia cells. Leuk Res 34: 1078-1082, 2010.

17. Zhao X, Yang L and Hu J: Down-regulation of miR-27a might inhibit proliferation and drug resistance of gastric cancer cells. J Exp Clin Cancer Res 30: 55, 2011.

18. Zhu W, Shan X, Wang T, Shu Y and Liu P: miR-181b modulates multidrug resistance by targeting BCL2 in human cancer cell lines. Int J Cancer 127: 2520-2529, 2010.

19. Akao Y, Noguchi S, Iio A, Kojima K, Takagi T and Naoe T: Dysregulation of microRNA-34a expression causes drugresistance to 5-FU in human colon cancer DLD-1 cells. Cancer Lett 300: 197-204, 2011.

20. Bai H, Xu R, Cao Z, Wei D and Wang C: Involvement of miR-21 in resistance to daunorubicin by regulating PTEN expression in the leukaemia K562 cell line. FEBS Lett 585: 402-408, 2011.

21. Yang H, Kong W, He L, et al: MicroRNA expression profiling in human ovarian cancer: miR-214 induces cell survival and cisplatin resistance by targeting PTEN. Cancer Res 68: 425-433, 2008.

22. Pan YZ, Morris ME and Yu AM: MicroRNA-328 negatively regulates the expression of breast cancer resistance protein (BCRP/ABCG2) in human cancer cells. Mol Pharmacol 75: 1374-1379, 2009.

23. Zhong X, Xiong M, Meng X and Gong R: Comparison of the multi-drug resistant human hepatocellular carcinoma cell line Bel-7402/ADM model established by three methods. J Exp Clin Cancer Res 29: 115, 2010.

24. Hummel R, Hussey DJ and Haier J: MicroRNAs: predictors and modifiers of chemo- and radiotherapy in different tumour types. Eur J Cancer 46: 298-311, 2010.

25. Sorrentino A, Liu CG, Addario A, Peschle C, Scambia G and Ferlini C: Role of microRNAs in drug-resistant ovarian cancer cells. Gynecol Oncol 111: 478-486, 2008. 
26. Galluzzi L, Morselli E, Vitale I, et al: miR-181a and miR-630 regulate cisplatin-induced cancer cell death. Cancer Res 70: 1793-1803, 2010.

27. Dai Y, Xie CH, Neis JP, Fan CY, Vural E and Spring PM: MicroRNA expression profiles of head and neck squamous cell carcinoma with docetaxel-induced multidrug resistance. Head Neck 33: 786-791, 2011.

28. Yu ZW, Zhong LP, Ji T, Zhang P, Chen WT and Zhang CP: MicroRNAs contribute to the chemoresistance of cisplatin in tongue squamous cell carcinoma lines. Oral Oncol 46: 317-322, 2010.

29. Boni V, Bitarte N, Cristobal I, et al: $\mathrm{miR}-192 / \mathrm{miR}-215$ influence 5-fluorouracil resistance through cell cycle-mediated mechanisms complementary to its post-transcriptional thymidilate synthase regulation. Mol Cancer Ther 9: 2265-2275, 2010.

30. Pogribny IP, Filkowski JN, Tryndyak VP, Golubov A, Shpyleva SI and Kovalchuk O: Alterations of microRNAs and their targets are associated with acquired resistance of MCF-7 breast cancer cells to cisplatin. Int J Cancer 127: 1785-1794, 2010.

31. Chang CJ, Hsu CC, Chang CH, et al: Let-7d functions as novel regulator of epithelial-mesenchymal transition and chemoresistant property in oral cancer. Oncol Rep 26: 1003-1010, 2011.

32. Salter KH, Acharya CR, Walters KS, et al: An integrated approach to the prediction of chemotherapeutic response in patients with breast cancer. PLoS One 3: e1908, 2008.

33. Chen L, Li H, Han L, et al: Expression and function of miR-27b in human glioma. Oncol Rep 26: 1617-1621, 2011.

34. Ji J, Zhang J, Huang G, Qian J, Wang X and Mei S: Overexpressed microRNA-27a and $27 \mathrm{~b}$ influence fat accumulation and cell proliferation during rat hepatic stellate cell activation. FEBS Lett 583: 759-766, 2009.

35. Lee JJ, Drakaki A, Iliopoulos D and Struhl K: MiR-27b targets PPARgamma to inhibit growth, tumor progression and the inflammatory response in neuroblastoma cells. Oncogene 31 : $3818-3825,2012$
36. Wang Y, Rathinam R, Walch A and Alahari SK: ST14 (suppression of tumorigenicity 14) gene is a target for miR-27b, and the inhibitory effect of ST14 on cell growth is independent of miR-27b regulation. J Biol Chem 284: 23094-23106, 2009.

37. Bhaumik D, Scott GK, Schokrpur S, et al: MicroRNAs $\mathrm{miR}-146 \mathrm{a} / \mathrm{b}$ negatively modulate the senescence-associated inflammatory mediators IL-6 and IL-8. Aging (Albany, NY) 1: 402-411, 2009.

38. Chatzikyriakidou A, Voulgari PV, Georgiou I and Drosos AA: The role of microRNA-146a (miR-146a) and its target IL-1Rassociated kinase (IRAK1) in psoriatic arthritis susceptibility. Scand J Immunol 71: 382-385, 2010.

39. Bhaumik D, Scott GK, Schokrpur S, Patil CK, Campisi J and Benz CC: Expression of microRNA-146 suppresses NF-kappaB activity with reduction of metastatic potential in breast cancer cells. Oncogene 27: 5643-5647, 2008.

40. Hurst DR, Edmonds MD, Scott GK, Benz CC, Vaidya KS and Welch DR: Breast cancer metastasis suppressor 1 up-regulates miR-146, which suppresses breast cancer metastasis. Cancer Res 69: 1279-1283, 2009.

41. Kogo R, Mimori K, Tanaka F, Komune S and Mori M: Clinical significance of miR-146a in gastric cancer cases. Clin Cancer Res 17: 4277-4284, 2011.

42. Gao W, Yu Y, Cao H, et al: Deregulated expression of miR-21, miR-143 and miR-181a in non small cell lung cancer is related to clinicopathologic characteristics or patient prognosis. Biomed Pharmacother 64: 399-408, 2010.

43. Schwind S, Maharry K, Radmacher MD, et al: Prognostic significance of expression of a single microRNA, miR-181a, in cytogenetically normal acute myeloid leukemia: a Cancer and Leukemia Group B study. J Clin Oncol 28: 5257-5264, 2010.

44. Wang XF, Shi ZM, Wang XR, et al: MiR-181d acts as a tumor suppressor in glioma by targeting K-ras and Bcl-2. J Cancer Res Clin Oncol 138: 573-584, 2012. 